\title{
The Co-Existence of Rheumatoid Arthritis and Systemic Lupus Erythematosus Biomarkers: Is It Rhupus?
}

\section{Type of article: conference abstract}

Y. Bouchedoub ${ }^{1,2}$, K. Salah_, ${ }_{-}^{1,2}$ N. Rachedi ${ }^{1,2}$, R. Babasaci ${ }^{1,2}$, L. Ould Ali ${ }^{1,2}$, R. Kherrache $^{1,2}$, M. Kherbeche ${ }^{1}$, A. Khlifati ${ }^{1}$, F/.Z. Guernou ${ }^{1}$, A. Benzitouni ${ }^{1}$, M. Semmana ${ }^{1}$, A. Meghlaoui ${ }^{1,2}$

${ }^{1}$ Immunology Laboratory, CHU Blida, Algeria

${ }^{2}$ Department of Medicine, Faculty of Medicine, Blida University, Algeria Corresponding Author: khadidjaslh4@gmail.com

\begin{abstract}
:
Background: Rhupus is a rare clinical condition where rheumatoid arthritis (RA) and systemic lupus erythematosus (SLE) overlap and is characterized by the presence of erosive arthritis with symptoms and signs of SLE. This study aims to investigate the prevalence of anti-CCP antibodies in SLE patients from CHU BLIDA (Immunology unit) and its association with anti-DNA and Anti Sm, in order to make a diagnosis of rhupus among our patients.

Methods: Our retrospective study included 96 patients fulfilling the American College of Rheumatology (ACR) classification criteria for lupus. anti-CCP antibodies, anti-Sm were analyzed by ELISA, anti-DNA antibodies were determined by both IFI on Crithidia luciliae substrate and ELISA. The FR by Laser Nephelemetry. Inclusion criteria are the presence of at least one immunological marker of LES with anti-CCP. The sex ratio $\mathrm{F} / \mathrm{H}$ is equal to $13 / 1$, where the average age is 37 years.

Results: Anti-CCP was found in 14 patients (14.6\% ), 56.25\% and $39.59 \%$ had positive anti-DNA and antiSm respectively; rheumatoid factors (RF) were positive in $27.08 \%$ of cases; anti-CCP / FR combination was found in $7.3 \%$ of cases. Besides, the combination of anti-CCP and anti-DNA was found in $12.5 \%$. These two autoantibodies were simultaneously absent in $49.92 \%$ of cases. Arthritis was found in 80 patients. Our results concerning the prevalence of immunological and clinical markers of RA such as anti-CCP, RF and arthritis in our lupus patients corroborate with those of the literature.

Conclusion: Based on the presence of shared clinical features of RA and SLE along with the presence of anti-DNA and anti-CCP antibodies in our patients, our findings strongly support the contention that rhupus is a true overlap between RA and SLE. Despite being a rare entity, it is important to know the clinical and humoral elements that allow its early diagnosis, making it easier to start treatment in a timely manner and reduce its possible complications.
\end{abstract}

Keywords: Rhupus, Anti CCP, FR, Anti-DNA, Anti-Sm, PR, LES.

\section{Declaration of conflicts}

This article is a conference abstract selected from the abstract book of the International Congress on Health Sciences and Medical Technologies ICHSMT'19, Tlemcen, Algeria, December 05-07, 2019. 
Medical Technologies Journal, Volume: 3, Issue: 4, October-December 2019, Pages: 489-490. Doi :

https://doi.org/10.26415/2572-004X-vol3iss4p489-490

\section{Authors' Biography}

No biography

\section{References}

No references 